\title{
UNITS IN MODULAR GROUP RINGS
}

\author{
D. B. COLEMAN AND D. S. PASSMAN
}

Abstract. Let $G$ be a finite p-group and let $U(G)$ denote the group of normalized units in the modular group ring of $G$. If $G$ is nonabelian, then it is shown that the nonregular group $\left.Z_{p}\right\rangle Z_{p}$ is involved in $U(G)$. Here $Z_{p}$ is the group with pelements and $Z_{p}\left\langle Z_{p}\right.$ is wreath product.

Let $F[G]$ denote the group ring of a finite $p$-group $G$ over the prime field $F=G F(p)$. We define the group of normalized units in $F[G]$ by

$$
U(G)=\left\{\sum_{x \in G} a_{x} \cdot x \in F[G] \mid \sum a_{x}=1\right\} .
$$

We see easily that $|U(G)|=p^{|G|-1}$ and that $U(G)$ is in fact a normal Sylow $p$-subgroup of the full group of units in $F[G]$. If $G$ is abelian, then $U(G)$ is abelian and its structure is therefore basically simple. On the other hand if $G$ is nonabelian then the structure of $U(G)$ becomes immediately very complicated. As an indication of this fact we offer the following results.

TheOREM. If $G$ is nonabelian, then $Z_{p} \backslash Z_{p}$ is involved in $U(G)$.

Corollary. If $G$ is nonabelian, then $U(G)$ is not regular.

The corollary follows immediately from the theorem since regularity is inherited by subgroups and quotient groups and since $Z_{p} \backslash Z_{p}$ is known to be nonregular. We remark that the corollary is trivial for $p=2$ since a 2 -group is regular if and only if it is abelian. On the other hand the proof of the theorem for $p=2$ requires the consideration of several special cases. For these, the following lemma is convenient. Here we let $o(x)$ denote the order of an element $x$.

Lemma. Let $x$ and $y$ be noncommuting elements of a 2-group $P$. Suppose that $o(x), o(y) \leqq 4$ and furthermore that if $o(x)=o(y)=4$ then $\langle x\rangle \cap\langle y\rangle=\langle 1\rangle$. Then $\left.Z_{2}\right\rangle Z_{2}$ is involved in $P$.

Proof. Clearly $Z_{2} \nmid Z_{2}$ is the dihedral group of order 8. Suppose first that $o(x)=o(y)=2$. If $u=x y$ then we have easily $u^{x}=u^{-1}$. Since $x$ and $u$ do not commute $o(u) \geqq 4$ and thus if $v$ is a suitable power of $u$

Presented to the Society January 24, 1968 under the title The unit group of $a$ modular group algebra; received by the editors July 28, 1969.

AMS Subject Classifications. Primary 2080, 2040.

Key Words and Phrases. p-group, group ring, group of units. 
with $o(v)=4$ then $\left.\langle v, x\rangle \cong Z_{2}\right\rangle Z_{2}$. Now suppose that $o(x)=2$ and $o(y)=4$. Since $x$ and $y$ do not commute we have $x \neq y^{2}$. By the above we can assume that $x$ and $y^{2}$ commute. If $(x, y)=y^{2}$ then clearly $\left.\langle x, y\rangle \cong Z_{2}\right\rangle Z_{2}$. On the other hand if $(x, y) \neq y^{2}$ then the images of $x$ and $y$ in $\langle x, y\rangle /\left\langle y^{2}\right\rangle$ are two noncommuting involutions so the result follows here. Finally let $o(x)=o(y)=4$ and $\langle x\rangle \cap\langle y\rangle=\langle 1\rangle$. We can clearly assume that $\left(x, y^{2}\right)=\left(y, x^{2}\right)=1$ so $x^{2}$ and $y^{2}$ are central in $\langle x, y\rangle$. Certainly $(x, y)$ cannot equal both $x^{2}$ and $y^{2}$ so if $(x, y) \neq x^{2}$ then $\langle x, y\rangle /\left\langle x^{2}\right\rangle$ contains two noncommuting elements one of order 2 and the other of order 4 . The result follows.

We now proceed to prove the theorem. There are three cases to consider, the first two being of a special nature.

Case 1. Suppose that all subgroups of $G$ are normal.

By Theorem 12.5.4 of [1] we have $p=2$ and $G=Q \times A$ where $Q$ is the quaternion group of order 8 and $A$ is elementary abelian. Let $Q=\langle x, y\rangle$ and consider $x$ and $u=1+x+y$ as elements of $U(G)$. We see easily that $o(x)=o(u)=4,\langle x\rangle \cap\langle u\rangle=\langle 1\rangle$ and $(x, u) \neq 1$. Thus by the lemma $Z_{2} \backslash Z_{2}$ is involved in $U(G)$.

Case 2. Suppose that $p=2$ and that $G$ has a nonnormal subgroup of order 2 .

Let $\langle x\rangle$ be the given nonnormal subgroup so $o(x)=2$ and $x$ is not central in $G$. Let $y \in G$ with $(x, y) \neq 1$. If $o(y) \leqq 4$ then by the lemma $Z_{2} \succ Z_{2}$ is involved in $G$ and hence in $U(G)$. Now let $o(y) \geqq 8$. Then there are four nonidentity elements of $\langle y\rangle$ having the same fourth power as $y$. Thus we can choose $z \in\langle y\rangle$ with $z^{4}=y^{4}$ and $z \neq 1, y, y^{x}$. Let $x$ and $u=1+y+z$ be elements of $U(G)$. Since $u^{x}=1+y^{x}+z^{x}$ we see by assumption that $(u, x) \neq 1$. Also since $y$ and $z$ commute and $y^{4}=z^{4}$ we have $u^{4}=1$. Thus $o(x)=2, o(u) \leqq 4$ so by the lemma $Z_{2} \backslash Z_{2}$ is involved in $U(G)$.

Case 3. Suppose finally that $G$ has a nonnormal subgroup and that if $p=2$ then this subgroup has order $\geqq 4$.

Let $A$ be a nonnormal subgroup of $G$ of maximal possible order and let $B$ be a subgroup with $A<B \subseteq G$ and $|B: A|=p$. Then $B \triangleleft G$ and all conjugates of $A$ are contained in $B$. Let $y \in G-N_{G}(A)$ with $y^{p} \in N_{G}(A)$ and set $A_{i}=A^{y^{i}}$ for $i=0,1, \cdots, p-1$. These are $p$ distinct subgroups of $B$ of index $p$. Hence $A_{i} \triangleleft B$.

Let $x_{i}=1+\hat{A}_{i}$ where $\hat{A}_{i}$ denotes the sum of the elements of $A_{i}$ in $F[G]$. Now if $i \neq j$ then $A_{i} A_{j}=B$ and hence since $p|| A_{i} \mid$ we have

$$
\begin{aligned}
\left(\dddot{A}_{i}\right)^{2} & =\left|A_{i}\right| A_{i}=0, & & \\
\left(A_{i}\right)\left(\dot{A}_{j}\right) & =\left|A_{i} \cap A_{j}\right| \hat{B}, & & \text { for } i \neq j, \\
\left(A_{i}\right)\left(A_{j}\right)\left(A_{k}\right) & =0, & & \text { for all } i, j, k .
\end{aligned}
$$


Note that for $i \neq j,\left|A_{i} \cap A_{j}\right|=|B| / p^{2}=|A| / p$. Let $X$ be the subgroup of $U(G)$ generated by the $x_{i}$. We show that $X$ is elementary abelian of order $p^{p}$.

By the above $X$ is clearly abelian. Also $x_{i}^{a_{i}}=1+a_{i} \hat{A}_{i}$ so $x_{i}^{p}=1$ and $X$ is elementary abelian. Now if $0 \leqq a_{i}<p$ then we have easily

$$
\prod x_{i}^{a_{i}}=1+\sum a_{i} \dot{A}_{i}+b \hat{B}
$$

where $b=(|A| / p) \sum_{i<j} a_{i} a_{j}$. Suppose that $\prod x_{i}^{a_{i}}=1$. Since $B>\cup A_{i}$ by order considerations it follows that the coefficient of any element of $B-\cup A_{i}$ in $\prod x_{i}^{a_{i}}$ is equal to $b$ and hence $b=0$. Then since $A_{i}>\cup_{j \neq i}\left(A_{j} \cap A_{i}\right)$ we see that $a_{i}=0$. This implies that $|X|=p^{p}$.

Now $x_{i}^{y}=x_{i+1}$ (subscripts modulo $p$ ) so $y \in U(G)$ normalizes $X$. Set $Y=\langle X, y\rangle$. We show that this is a split extension, that is, that $X \cap\langle y\rangle=\langle 1\rangle$. Let $w \in X \cap\langle y\rangle$. Since $w \in\langle y\rangle$ we have $w \in G$. Clearly $y$ centralizes $w$ so we must have $w=\prod x_{i}^{a}$ for some $a$. Thus by the above

$$
w=\prod \stackrel{a}{x_{i}}=1+\sum a A_{i}+b \hat{B}
$$

where $b=(|A| / p)(p(p-1) / 2) a^{2}$. If $p>2$ then $p(p-1) / 2$ is zero in $F$ while if $p=2$ then $|A| / p$ is zero in $F$ by assumption. Hence in either case $b=0$. The coefficient of the identity in the above right-hand expression is then $1+p a=1$ so since $w \in G$ we have $w=1$.

Finally $y^{p}$ is clearly central in $Y$ and since $\left\langle y^{p}\right\rangle \cap X=\langle 1\rangle$ we have $\left.Y /\left\langle y^{p}\right\rangle \cong Z_{p}\right\rangle Z_{p}$ is involved in $U(G)$. This completes the proof of the theorem.

\section{REFERENCE}

1. M. Hall, Jr., The theory of groups, Macmillan, New York, 1959. MR 21 \#1996.

University of Kentucky, Lexington, Kentucky 40506 and UNIVERSity OF Wisconsin, Madison, Wisconsin 53706 